Saisho, $\mathbf{Y}$. and Tanaka, $H$.

Osaka J. Math.

23 (1986), 725-740

\title{
STOCHASTIC DIFFERENTIAL EQUATIONS FOR MUTUALLY REFLECTING BROWNIAN BALLS
}

\author{
Yasumasa SAISHO aNd Hiroshi TANAKA
}

(Received August 1, 1985)

\section{Introduction}

In this paper we construct a random motion of mutually reflecting hard balls of diameter $\rho$ in $\boldsymbol{R}^{d}$ by solving certain stochastic differential equation (abbreviated: SDE) with a kind of singular drift. For simplicity we first consider the motion of mutually reflecting Brownian balls of diameter $\rho$. In order to construct such a motion we pose the following problem. Let $W$ denote the space of continuous paths in $\boldsymbol{R}^{d}$. Given $w_{1}, \cdots, w_{n} \in W$ satisfying

$$
\left|w_{i}(0)-w_{j}(0)\right| \geqq \rho, \quad 1 \leqq i<j \leqq n,
$$

solve the equation

$$
\xi_{i}(t)=w_{i}(t)+\sum_{j=1(\neq i)}^{n} \int_{0}^{t}\left(\xi_{i}(s)-\xi_{j}(s)\right) d \phi_{i j}(s), \quad 1 \leqq i \leqq n,
$$

under the following conditions (3) and (4).

(3) $\xi_{i} \in W, 1 \leqq i \leqq n$, and $\left|\xi_{i}(t)-\xi_{j}(t)\right| \geqq \rho, 1 \leqq i<j \leqq n, t \geqq 0$.

(4) $\phi_{i j}$ 's are continuous non-decreasing functions with $\phi_{i j}(0)=0, \phi_{i j}(t)=\phi_{j i}(t)$ and

$$
\phi_{i j}(t)=\int_{0}^{t} 1_{\rho}\left(\left|\xi_{i}(s)-\xi_{j}(s)\right|\right) d \phi_{i j}(s),
$$

where $\mathbf{1}_{\rho}(r)=1$ if $r=\rho$, and $=0$ if $r \neq \rho$.

A pair $(\xi, \phi)$ of functions or simply a function $\xi$ is called a solution of (2) provided that (2), (3) and (4) are satisfied. One of the main results in this paper is that there exists a unique solution of (2) for given $w_{1}, \cdots, w_{n}$. By taking $w_{1}, \cdots, w_{n}$ to be independent $d$-dimensional Brownian motions satisfying (1), we obtain a process $\left(\xi_{1}(t), \cdots, \xi_{n}(t)\right)$. This is what we call the motion of mutually reflecting Brownian balls. $\xi_{i}(t)$ denotes the center of the $i$-th Brownian ball at time $t$. In analogy with Skorohod's equation for a 1-dimensional reflecting Brownian motion ([3] [5] [7]), the equation (2) may be regarded as Skorohod's 
equation for the motion of mutually reflecting Brownian balls.

We next consider the $\operatorname{SDE}$ on a probability space $(\Omega, \mathscr{F}, P)$ :

$$
\begin{aligned}
& d X_{i}(t)=\sigma\left(X_{i}(t)\right) d B_{i}(t)+b\left(X_{i}(t)\right) d t \\
& \quad+\sum_{j=1(\neq i)}^{n}\left(X_{i}(t)-X_{j}(t)\right) d \phi_{i j}(t), \quad 1 \leqq i \leqq n,
\end{aligned}
$$

where

$$
\sigma: \boldsymbol{R}^{d} \rightarrow \boldsymbol{R}^{d} \otimes \boldsymbol{R}^{d}, \quad b: \boldsymbol{R}^{d} \rightarrow \boldsymbol{R}^{d}
$$

are given, $X_{i}(0)$ 's are $\mathscr{F}_{0}$-measurable initial values satisfying $\left|X_{i}(0)--X_{j}(0)\right| \geqq \rho$, $1 \leqq i<j \leqq n$, and $B_{i}(t), 1 \leqq i \leqq n$, are independent $d$-dimensional $\mathscr{F}_{t}$-adapted Brownian motion with $B_{i}(0)=0$. Here $\left\{\mathscr{F}_{t}\right\}_{t \geqq 0}$ is a right continuous filtration on $(\Omega, \mathscr{F}, P)$ such that each $\mathscr{F}_{t}$ contains all $P$-negligible sets. As in $(2), X_{i}(t)$ and $\Phi_{i j}(t)$ should be found under the following conditions (6) and (7).

(6) $X_{i}(t)$ 's are $\mathscr{F}_{t}$-adapted continuous processes with $\left|X_{i}(t)-X_{j}(t)\right| \geqq \rho, 1 \leqq i<$ $j \leqq n, t \geqq 0$.

(7) $\Phi_{i j}(t)$ 's are $\mathscr{F}_{t}$-adapted continuous non-decreasing processes with $\Phi_{i j}(0)=$ $0, \Phi_{i j}(t)=\Phi_{j i}(t)$ and

$$
\Phi_{i j}(t)=\int_{0}^{t} 1_{\rho}\left(\left|X_{i}(s)-X_{j}(s)\right|\right) d \Phi_{i j}(s)
$$

The equation (5) may be considered as Skorohod's SDE for mutually reflecting diffusion balls with coefficients $\sigma$ and $b$. Another main result of this paper is that there exists a unique solution of (5) provided that $\sigma$ and $b$ are bounded and Lipschitz continuous.

Our method for solving (2) and (5) is to make use of the results of [6] concerning Skorohod's equation for general domain. Skorohod's equation for a multi-dimensional domain $D$ with reflecting boundary (a precise formulation is explained in 1) was discussed by Tanaka [8] when $D$ is a convex domain and then by Lions and Sznitman [4] when $D$ is a general domain satisfying Conditions (A) and (B) (see 1) together with the additional condition that $D$ is admissible, which means roughly that $D$ can be approximated in some sense by smooth domains. Recently, Frankowska [2] and Saisho [6] amplified Lions and Sznitman's result by removing the additional condition. Now the present discussion is based on the fact that Skorohod's equation (2) (or SDE (5)) is equivalent to Skorohod's equation (or SDE) for the domain

$$
D=\left\{\left(x_{1}, \cdots, x_{n}\right) \in \boldsymbol{R}^{n d}:\left|x_{i}-x_{j}\right|>\rho, 1 \leqq i<j \leqq n\right\} .
$$

So the crucial point of our discussions is to prove that the domain $D$ of (8) satisfies Conditions (A) and (B). In solving Skorohod's SDE for $D$ we make 
use of Theorem 5.1 of Saisho [6].

In 1 we state briefly the results of Lions and Sznitman [4] and Saisho [6] concerning Skorohod's equation for a general domain. We prove that the domain $D$ of (8) satisfies Condition (B) in 2 and (A) in 3. We solve Skorohod's equation (2) in 4 and $\operatorname{SDE}(5)$ in 5.

1. Some known results on Skorohod's equation for an $N$-dimensional domain with reflecting boundary

Let $D$ be a domain in $\boldsymbol{R}^{N}$ and define the set $\Re_{x}$ of inward normal unit vectors at $\boldsymbol{x} \in \partial D$ by

$$
\begin{aligned}
& \mathscr{N}_{\boldsymbol{x}}=\bigcup_{r>0} \mathscr{I}_{x, r}, \\
& \mathscr{N}_{x, r}=\left\{\boldsymbol{n} \in \boldsymbol{R}^{N}:|\boldsymbol{n}|=1, B(\boldsymbol{x}-\boldsymbol{r n}, r) \cap D=\phi\right\},
\end{aligned}
$$

where $B(\boldsymbol{z}, r)=\left\{\boldsymbol{y} \in \boldsymbol{R}^{N}:|\boldsymbol{y}-\boldsymbol{z}|<r\right\}, \boldsymbol{z} \in \boldsymbol{R}^{N}$. In general it can happen that $\eta_{\boldsymbol{x}}=\phi$. In what follows $\langle\cdot, \cdot\rangle$ denotes the usual inner product in $\boldsymbol{R}^{N}$. We introduce two conditions on the domain $D$.

Condition $(A)$ (uniform exterior sphere condition). There exists a constant $r_{0}>0$ such that

$$
\Re_{x}=\mathscr{I}_{x, r_{0}} \neq \phi \text { for any } \boldsymbol{x} \in \partial D \text {. }
$$

Condition $(B)$. There exist constants $\delta>0$ and $\beta(1 \leqq \beta<\infty)$ with the following property: for any $\boldsymbol{x} \in \partial D$ there exists a unit vector $\boldsymbol{l}_{\boldsymbol{x}}$ such that

$$
\left\langle\boldsymbol{l}_{\boldsymbol{x}}, \boldsymbol{n}\right\rangle \geqq 1 / \beta \quad \text { for any } n \in \underset{\boldsymbol{y} \in B(x, \delta) \cap \partial D}{\cup} \Re_{y} .
$$

REMARK 1.1. For any fixed $r>0$ and a unit vector $\boldsymbol{n}$ the following two statements are equivalent.

(i) $B(\boldsymbol{x}-\boldsymbol{r n}, r) \cap D=\phi$.

(ii) $\langle\boldsymbol{y}-\boldsymbol{x}, \boldsymbol{n}\rangle+\frac{1}{2 r}|\boldsymbol{y}-\boldsymbol{x}|^{2} \geqq 0$ for any $\boldsymbol{y} \in \bar{D}$.

Remark 1.2. $D$ satisfies Condition (B) if it satisfies the following condition.

Condition $\left(B^{\prime}\right)$. There exist $\delta>0$ and $\alpha(0 \leqq \alpha<1)$ with the following property: for any $\boldsymbol{x} \in \partial D$ there exists a unit vector $\boldsymbol{l}_{\boldsymbol{x}}$ such that

$$
C\left(\boldsymbol{y}, \boldsymbol{l}_{\boldsymbol{x}}, \alpha\right) \cap B(\boldsymbol{x}, \delta) \subset \bar{D}, \quad \forall \boldsymbol{y} \in B(\boldsymbol{x}, \delta) \cap \partial D,
$$

where $C\left(\boldsymbol{y}, \boldsymbol{l}_{\boldsymbol{x}}, \alpha\right)$ is the convex cone with vertex $\boldsymbol{y}$, defined by 


$$
C\left(\boldsymbol{y}, \boldsymbol{l}_{\boldsymbol{x}}, \alpha\right)=\left\{\boldsymbol{z} \in \boldsymbol{R}^{N}:\left\langle z-\boldsymbol{y}, \boldsymbol{l}_{\boldsymbol{x}}\right\rangle \geqq \alpha|z-\boldsymbol{y}|\right\} .
$$

Denote by $W\left(\boldsymbol{R}^{N}\right)$ (resp. $W(\bar{D})$ ) the space of continuous paths in $\boldsymbol{R}^{N}$ (resp. $\bar{D})$. Skorohod's equation for $D$ with reflecting boundary is written in the form

$$
\xi(t)=w(t)+\int_{0}^{t} n(s) d \phi(s),
$$

where $w \in W\left(\boldsymbol{R}^{N}\right)$ is given and satisfies $w(0) \in \bar{D}$; a solution $(\xi, \phi)$ of $(1.1)$ should be found under the following conditions.

(1.2) $\xi \in W(\bar{D})$.

(1.3) $\phi$ is a continuous non-decreasing function such that $\phi(0)=0$ and

$$
\phi(t)=\int_{0}^{t} \mathbf{1}_{\partial D}(\xi(s)) d \phi(s) .
$$

(1.4) $\boldsymbol{n}(s) \in \mathscr{N}_{\xi(s)}$ if $\xi(s) \in \partial D$.

The following theorem was proved by Lions and Sznitman [4] under the additional condition that $D$ is admissible. Frankowska [2] and Saisho [6] removed this additional condition. Frankowska's result is of a general type but contains what we need only in a less explicit form, so we state the theorem in the form of Saisho [6].

Theorem 1.1. If the domain $D$ satisfies Conditions $(A)$ and $(B)$, then there exists a unique solution of (1.1) for any given $w \in W\left(\boldsymbol{R}^{N}\right)$ with $w(0) \in \bar{D}$.

Next, given

$$
\sigma: \bar{D} \rightarrow \boldsymbol{R}^{N} \otimes \boldsymbol{R}^{N}, \quad b: \bar{D} \rightarrow \boldsymbol{R}^{N},
$$

we consider Skorohod's SDE

$$
d X(t)=\sigma(X(t)) d B(t)+b(X(t)) d t+\boldsymbol{n}(t) d \Phi(t),
$$

where the initial value $X(0) \in \bar{D}$ is assumed to be $\mathscr{F}_{0}$-measurable and $B(\imath)$ is an $\boldsymbol{N}$-dimensional $\mathscr{F}_{t}$-adapted Brownian motion with $B(0)=0$. Here $\left\{\mathscr{F}_{t}\right\}$ is a right continuous filtration on $(\Omega, \mathscr{F}, P)$ such that $\mathscr{F}_{0}$ contains all $P$-negligible sets. A solution $(X(t), \Phi(t))$ shculd be found under the following conditions (1.6)-(1.8).

(1.6) $\quad X(t)$ is a $\bar{D}$-valued $\mathscr{F}_{t}$-adapted continuous process.

(1.7) $\Phi(t)$ is a continuous non-decreasing process with $\Phi(0)=0$ and

$$
\Phi(t)=\int_{0}^{t} \mathbf{1}_{\partial D}(X(s)) d \Phi(s)
$$


(1.8) $\boldsymbol{n}(s) \in \mathscr{T}_{X(s)} \quad$ if $\quad X(s) \in \partial D$

In addition to Conditions (A) and (B), Lions and Sznitman [4] introduced the following Condition (C) and discussed the existence and uniqueness of the solution of (1.5).

Condition $(C)$. There exists a function $f$ in $C^{2}\left(\boldsymbol{R}^{N}\right)$ which is bounded together with its first and second partial derivatives such that $\exists \gamma>0, \forall \boldsymbol{x} \in \partial D$, $\forall \boldsymbol{y} \in \bar{D}, \forall \boldsymbol{n} \in \Re_{\boldsymbol{x}}$

$$
\langle\boldsymbol{y}-\boldsymbol{x}, \boldsymbol{n}\rangle+\frac{1}{\gamma}\langle\nabla f(\boldsymbol{x}), \boldsymbol{n}\rangle|\boldsymbol{y}-\boldsymbol{x}|^{2} \geqq 0 .
$$

The following theorem was proved by Lions and Sznitman ([4: Theorem 3.1]) under Condition (C) and the admissibility of $D$; however, recently Saisho ([6: Theorem 5.1]) removed these additional conditions.

Theorem 1.2 ([6]). Let D satisfy Conditions $(A),(B)$ and assume that $\sigma$ and $b$ are bounded and Lipschitz continuous. Then for any initial value $X(0) \in \bar{D}$ there exists a unique (strong) solution of (1.5).

\section{D satisfies Condition $\left(B^{\prime}\right)$}

Let $D$ be the domain in $\boldsymbol{R}^{n d}$ defined by (8). We are going to prove the following proposition.

Proposition 2.1. The domain $D$ satisfies Condition $\left(B^{\prime}\right)$, that is, there exist constants $\delta>0$ and $\alpha(0 \leqq \alpha<1)$ with the following property: for any $\boldsymbol{x} \in \partial D$ there exists a unit vector $\boldsymbol{l}_{\boldsymbol{x}}$ such that

$$
C\left(\boldsymbol{y}, \boldsymbol{l}_{\boldsymbol{x}}, \alpha\right) \cap B(\boldsymbol{x}, \delta) \subset \bar{D}
$$

holds for any $\boldsymbol{y} \in B(\boldsymbol{x}, \delta) \cap \partial D$.

For a non-empty subset $I$ of $\{1,2, \cdots, n\}$ and $\boldsymbol{x}=\left(x_{1}, \cdots, x_{n}\right) \in \bar{D}$ we set

$$
\boldsymbol{x}(I)=\left\{x_{i}: i \in I\right\} .
$$

Definition 2.1. Let $I, I^{\prime}$ be non-empty subsets of $\{1,2, \cdots, n\}$.

(i) $\boldsymbol{x}(I)$ and $\boldsymbol{x}\left(I^{\prime}\right)$ are said to be separated if

$$
I \cap I^{\prime}=\phi \text { and }\left|x_{i}-x_{j}\right| \geqq 2 \rho, \forall i \in I, \forall j \in I^{\prime} .
$$

(ii) $\boldsymbol{x}(I)$ is called a cluster if

(2.3) for any $i, j \in I$ with $i \neq j$ there exist $i_{0}(=i), i_{1}, \cdots, i_{p-1}, i_{p}(=j)$ in $I$ such that $\left|x_{i_{k-1}}-x_{i_{k}}\right|<2 \rho, 1 \leqq k \leqq p$. 
REMARK 2.1. If $\boldsymbol{x}(I)$ is a cluster, then

$$
\begin{aligned}
\left|x_{i}-x_{j}\right| & <2(n-1) \rho, \quad \forall i, j \in I, \\
\left|x_{i}-x_{I}\right| & =\frac{1}{\sharp I}\left|\sum_{j \in I}\left(x_{i}-x_{j}\right)\right| \\
& <2(n-1) \rho, \quad \forall i \in I,
\end{aligned}
$$

where $x_{I}=(\sharp I)^{-1} \sum_{j \in I} x_{j}$ and $\sharp I$ is the number of elements in $I$.

Let $\boldsymbol{x}=\left(x_{1}, \cdots, x_{n}\right)$. Then $\left\{x_{1}, \cdots, x_{n}\right\}$ can be represented as the sum of mutually separated clusters:

$$
\left\{x_{1}, \cdots, x_{n}\right\}=\bigcup_{k=1}^{m} \boldsymbol{x}\left(I_{k}\right) .
$$

Here $1 \leqq m \leqq n$. In what follows we assume that $\boldsymbol{x} \in \partial D$ and keep it fixed, so $I_{1}, \cdots, I_{m}$ appearing in (2.6) are also fixed. Let $c>1$ be a constant which will be determined later and set

$$
\left\{\begin{array}{l}
u_{i}=x_{I_{k}}+c\left(x_{i}-x_{I_{k}}\right), \quad i \in I_{k}, 1 \leqq k \leqq m \\
\boldsymbol{u}=\left(u_{1}, \cdots, u_{n}\right) \in \boldsymbol{R}^{n d}
\end{array}\right.
$$

Also we set for $\boldsymbol{y}=\left(y_{1}, \cdots, y_{n}\right) \in \boldsymbol{R}^{n d}$

$$
\left\{\begin{array}{l}
v_{i}=y_{I_{k}}+c\left(y_{i}-y_{I_{k}}\right), \quad i \in I_{k}, 1 \leqq k \leqq m, \\
\boldsymbol{v}=\left(v_{1}, \cdots, v_{n}\right) \in \boldsymbol{R}^{n d} .
\end{array}\right.
$$

We are going to prove Proposition 2.1 with

$$
\left\{\begin{array}{l}
\boldsymbol{l}_{\boldsymbol{x}}=\frac{\boldsymbol{u}-\boldsymbol{x}}{|\boldsymbol{u}-\boldsymbol{x}|}, \\
\delta=\frac{\varepsilon}{4(c-1)+2} \\
\alpha=\cos \theta
\end{array}\right.
$$

where $c, \varepsilon$ and $\theta$ are constants determined by

$$
\begin{gathered}
\left\{\begin{array}{l}
(c-1)(n-1)=1 / 8, \\
\varepsilon=(c-1) \rho / 4,
\end{array}\right. \\
\sin \frac{\theta}{2}=\frac{\varepsilon}{8(c-1) \sqrt{n}(n-1) \rho}, 0<\theta \leqq \pi / 2 .
\end{gathered}
$$

From now on let $c, \varepsilon, \delta$ be constants determined by (2.7) and (2.8). Let $\boldsymbol{y} \in$ $B(\boldsymbol{x}, \delta) \cap \partial D$ and set

$$
z=\boldsymbol{y}+(\boldsymbol{u}-\boldsymbol{x})
$$


Lemma 2.1. $|u-x|=|z-y|<2(c-1) \sqrt{n}(n-1) \rho$.

Proof. Since $i \in I_{k}$ for some $k$, we have

$$
\left|u_{i}-x_{i}\right|=(c-1)\left|x_{i}-x_{I_{k}}\right|<2(c-1)(n-1) \rho
$$

by (2.5), and hence we obtain the lemma.

Lemma 2.2. (i) For any $\boldsymbol{u}^{\prime} \in B(\boldsymbol{u}, \varepsilon), \boldsymbol{v}^{\prime} \in B(\boldsymbol{v}, \varepsilon)$ and $0<t \leqq 1$,

$$
u^{\prime \prime}=(1-t) x+t u^{\prime} \in D, \quad v^{\prime \prime}=(1-t) y+t v^{\prime} \in D .
$$

(ii) $B(\boldsymbol{u}, \varepsilon) \subset D, \quad B(\boldsymbol{v}, \varepsilon) \subset D$.

Proof. Since (ii) can be proved by setting $t=1$ in (i), it is enough to prove (i), that is,

$$
\left|u_{i}^{\prime \prime}-u_{j}^{\prime \prime}\right|>\rho, \quad\left|v_{i}^{\prime \prime}-v_{j}^{\prime \prime}\right|>\rho, \quad 1 \leqq i<j \leqq n .
$$

Case (I): $i, j \in I_{k}$ for some $k$. Since

$$
\begin{aligned}
u_{i}^{\prime \prime}-u_{j}^{\prime \prime} & =(1-t)\left(x_{i}-x_{j}\right)+t\left\{\left(u_{i}-u_{j}\right)+\left(u_{i}^{\prime}-u_{i}\right)-\left(u_{j}^{\prime}-u_{j}\right)\right\} \\
& =\{1+(c-1) t\}\left(x_{i}-x_{j}\right)+t\left\{\left(u_{i}^{\prime}-u_{i}\right)-\left(u_{j}^{\prime}-u_{j}\right)\right\},
\end{aligned}
$$

we have

$$
\left|u_{i}^{\prime \prime}-u_{j}^{\prime \prime}\right| \geqq\{1+(c-1) t\} \rho-2 \varepsilon t>\rho,
$$

and a similar inequality for $\left|v_{i}^{\prime \prime}-v_{j}^{\prime \prime}\right|$.

Case (II): $i \in I_{k}, j \in I_{l}(k \neq l)$. Since

$$
u_{i}^{\prime \prime}-x_{i}=t\left(u_{i}^{\prime}-x_{i}\right)=t\left(u_{i}-x_{i}\right)+t\left(u_{i}^{\prime}-u_{i}\right),
$$

we have

$$
\begin{aligned}
\left|u_{i}^{\prime \prime}-x_{i}\right| & \leqq\left|u_{i}-x_{i}\right|+\left|u_{i}^{\prime}-u_{i}\right| \quad(\text { setting } t=1) \\
& <2(c-1)(n-1) \rho+\varepsilon \quad(\text { by }(2.10)),
\end{aligned}
$$

and hence, making use of the inequality $\left|x_{i}-x_{j}\right| \geqq 2 \rho$ which is a consequence of the assumption that $\boldsymbol{x}\left(I_{k}\right)$ and $\boldsymbol{x}\left(I_{l}\right)$ are separated, we have

$$
\begin{aligned}
\left|u_{i}^{\prime \prime}-u_{j}^{\prime \prime}\right| & \geqq\left|x_{i}-x_{j}\right|-\left|u_{i}^{\prime \prime}-x_{i}\right|-\left|u_{j}^{\prime \prime}-x_{j}\right| \\
& >2 \rho-4(c-1)(n-1) \rho-2 \varepsilon>\rho .
\end{aligned}
$$

Next, to prove the inequality for $\left|v_{i}^{\prime \prime}-v_{j}^{\prime \prime}\right|$, we notice that $\approx$

$$
\begin{aligned}
& \left|\left(y_{i}-y_{I_{k}}\right)-\left(x_{i}-x_{I_{k}}\right)\right|=\left|\left(y_{i}-x_{i}\right)-\frac{1}{\sharp I_{k}} \sum_{p \in I_{k}}\left(y_{p}-x_{p}\right)\right| \\
& \quad \leqq 2 \max _{1 \leqq p \leqq n}\left|y_{p}-x_{p}\right| \leqq 2|\boldsymbol{y}-\boldsymbol{x}|<2 \delta,
\end{aligned}
$$


from which it follows that

$$
\left|y_{i}-y_{I_{k}}\right|<\left|x_{i}-x_{I_{k}}\right|+2 \delta<2(n-1) \rho+2 \delta \quad \text { (use (2.5)). }
$$

Therefore

$$
\left|v_{i}-y_{i}\right|=(c-1)\left|y_{i}-y_{I_{k}}\right|<2(c-1)(n-1) \rho+2(c-1) \delta,
$$

and hence

$$
\left|v_{i}^{\prime \prime}-y_{i}\right| \leqq t\left|v_{i}-y_{i}\right|+t\left|v_{i}^{\prime}-v_{i}\right|<2(c-1)(n-1) \rho+2(c-1) \delta+\varepsilon .
$$

Thus we have

$$
\begin{aligned}
& \left|v_{i}^{\prime \prime}-v_{j}^{\prime \prime}\right| \geqq\left|y_{i}-y_{j}\right|-\left|v_{i}^{\prime \prime}-y_{i}\right|-\left|v_{j}^{\prime \prime}-y_{j}\right| \\
& \quad \geqq\left|x_{i}-x_{j}\right|-\left|y_{i}-x_{i}\right|-\left|y_{j}-x_{j}\right|-\left|v_{i}^{\prime \prime}-y_{i}\right|-\left|v_{j}^{\prime \prime}-y_{j}\right| \\
& \quad>2 \rho-2 \delta-4(c-1)(n-1) \rho-4(c-1) \delta-2 \varepsilon \\
& \quad>\rho .
\end{aligned}
$$

The proof of the lemma is finished.

Lemma 2.3. $|z-v|<\varepsilon / 2$.

Proof. Taking $I_{k}$ such that $i \in I_{k}$, we have

$$
\begin{aligned}
\left|z_{i}-v_{i}\right| & =\left|(c-1)\left(x_{i}-y_{i}\right)-(c-1)\left(x_{I_{k}}-y_{I_{k}}\right)\right| \\
& \leqq(c-1)\left|x_{i}-y_{i}\right|+(c-1)\left|x_{I_{k}}-y_{I_{k}}\right|,
\end{aligned}
$$

and hence

$$
\begin{aligned}
|z-\boldsymbol{v}|^{2} & =\sum_{i=1}^{n}\left|z_{i}-v_{i}\right|^{2} \\
& \leqq 2(c-1)^{2}|\boldsymbol{x}-\boldsymbol{y}|^{2}+2(c-1)^{2} \sum_{k=1}^{m} \sharp I_{k}\left|x_{I_{k}}-y_{I_{k}}\right|^{2} .
\end{aligned}
$$

Because $\left|x_{I_{k}}-y_{I_{k}}\right|^{2} \leqq\left(\# I_{k}\right)^{-1} \sum_{j \in I_{k}}\left|x_{j}-y_{j}\right|^{2}$, we have

$$
\sum_{k=1}^{m} \sharp I_{k}\left|x_{I_{k}}-y_{I_{k}}\right|^{2} \leqq|\boldsymbol{x}-\boldsymbol{y}|^{2},
$$

and (2.11) yields

$$
|z-\boldsymbol{v}| \leqq 2(c-1)|x-y|<2(c-1) \delta<\varepsilon / 2 .
$$

The proof of the lemma is finished.

Proof of Proposition 2.1. By (ii) of Lemma 2.2 and Lemma 2.3 we have

$$
B(z, \varepsilon / 2) \subset B(v, \varepsilon) \subset D,
$$

and hence by (i) of Lemma 2.2 


$$
\bar{D} \supset \text { the convex hull } \Gamma \text { of the set } B(z, \varepsilon / 2) \cup\{y\} \text {. }
$$

We have also

$$
|z-y|=|u-x| \geqq \varepsilon>2 \delta,
$$

because $\boldsymbol{x} \notin B(\boldsymbol{u}, \varepsilon)$ by (ii) of Lemma 2.2. Therefore, if $\theta$ is defined by (2.9), then (2.12) combined with Lemma 2.1 and (2.13) implies that

$$
C\left(y, l_{x}, \cos \theta\right) \cap B(y, 2 \delta) \subset \bar{\Gamma} \subset \bar{D} .
$$

Since $B(\boldsymbol{x}, \delta) \subset B(\boldsymbol{y}, 2 \delta),(2.14)$ implies (2.1) with $\alpha=\cos \theta$. The proof of Proposition 2.1 is finished.

\section{D satisfies Condition (A)}

For $1 \leqq i<j \leqq n$ let $D_{i j}$ be the domain defined by

$$
D_{i j}=\left\{\boldsymbol{x}=\left(x_{1}, \cdots, x_{n}\right) \in \boldsymbol{R}^{n d}:\left|x_{i}-x_{j}\right|>\rho\right\} .
$$

The domain $D$ of (8) is expressed as

$$
D=\bigcap_{1 \leqq i<j \leqq n} D_{i j}
$$

and $\boldsymbol{x} \in \partial D$ implies $\boldsymbol{x} \in \bigcap_{(i, j) \in L} \partial D_{i j}$, where

$$
L=L_{x}=\left\{(i, j): 1 \leqq i<j \leqq n,\left|x_{i}-x_{j}\right|=\rho\right\} .
$$

Obviously, if $\boldsymbol{x} \in \bar{D}$ then $\boldsymbol{x} \in \partial D$ is equivalent to $L_{x} \neq \phi . \quad$ For $(i, j) \in L_{x}$ we define a unit vector $\boldsymbol{n}_{i j}$ in $\boldsymbol{R}^{n d}$ by

$$
\boldsymbol{n}_{i j}=\left\{0, \cdots, 0, \frac{x_{i}-x_{j}}{\sqrt{2} \rho}, 0, \cdots, 0, \frac{x_{j}-x_{i}}{\sqrt{2} \rho}, 0, \cdots, 0\right\},
$$

let $\alpha(0 \leqq \alpha<1)$ be the constant appearing in Proposition 2.1 and set

$$
r_{0}=\rho \sqrt{\frac{1-\alpha^{2}}{2}} .
$$

Proposition 3.1. The domain $D$ satisfies Condition $(A)$ with $r_{0}=\rho \sqrt{\frac{1-\alpha^{2}}{2}}$, and for any $\boldsymbol{x} \in \partial D$

$$
\bigcap_{x}=\left\{n=\sum_{(i, j) \in L} c_{i j} n_{i j}: c_{i j} \geqq 0,|n|=1\right\} .
$$

Lemma 3.1. (i) $B\left(\boldsymbol{x}-2^{-1 / 2} \rho \boldsymbol{n}_{i j}, 2^{-1 / 2} \rho\right) \cap D_{i j}=\phi, x \in \partial D_{i j}$.

(ii) $B\left(\boldsymbol{x}-2^{-1 / 2} \rho \boldsymbol{n}_{i j}, 2^{-1 / 2} \rho\right) \cap D=\phi$ for any $(i, j) \in L_{x}, \boldsymbol{x} \in \partial D$, that is, $\boldsymbol{n}_{i j} \in$ 
$\Re_{x, 2^{-1 / 2_{p}}}$ for any $(i, j) \in L_{x}$.

We set

Proof. Since (ii) follows immediately from (i), it is enough to prove (i).

$$
\boldsymbol{y}=\boldsymbol{x}-2^{-1 / 2} \rho \boldsymbol{n}_{i j},
$$

that is,

$$
y_{k}= \begin{cases}\left(x_{i}+x_{j}\right) / 2 & \text { for } k=i, j \\ x_{k} & \text { for } k \neq i, j\end{cases}
$$

Then for any $z \in B\left(\boldsymbol{y}, 2^{-1 / 2} \rho\right)$ we have

$$
\begin{aligned}
\left|z_{i}-z_{j}\right| & \leqq\left|z_{i}-y_{i}\right|+\left|y_{i}-y_{j}\right|+\left|y_{j}-z_{j}\right| \\
& =\left|z_{i}-y_{i}\right|+\left|z_{j}-y_{j}\right| \leqq \sqrt{2}|z-\boldsymbol{y}|<\rho,
\end{aligned}
$$

and hence $z \notin D_{i j}$, completing the proof.

Lemma 3.2. Let $\Re_{x}^{\prime}$ be the right-hand side of (3.1). Then

$$
\mathfrak{N}_{x}^{\prime} \subset \mathfrak{N}_{x, r_{0}}, \quad \boldsymbol{x} \in \partial D .
$$

Proof. By Remark 1.1 it is enough to prove that for any $\boldsymbol{n} \in \Re_{x}^{\prime}$

$$
\langle\boldsymbol{y}-\boldsymbol{x}, \boldsymbol{n}\rangle+\frac{1}{2 r_{0}}|\boldsymbol{x}-\boldsymbol{y}|^{2} \geqq 0, \quad \forall \boldsymbol{y} \in \bar{D} .
$$

Let $\boldsymbol{l}=\boldsymbol{l}_{\boldsymbol{x}}$ be the unit vector appearing in Proposition 2.1. Then by Proposition 2.1

$$
C(\boldsymbol{x}, \boldsymbol{l}, \alpha) \cap B(\boldsymbol{x}, \delta) \subset \bar{D}
$$

from which it follows that

$$
\boldsymbol{x}-\Re_{\boldsymbol{x}} \subset C(\boldsymbol{x}, \boldsymbol{l}, \alpha)^{*},
$$

where $C(\boldsymbol{x}, \boldsymbol{l}, \alpha)^{*}$ is the dual cone of $C(\boldsymbol{x}, \boldsymbol{l}, \alpha)$, that is,

$$
\begin{aligned}
C(\boldsymbol{x}, \boldsymbol{l}, \alpha)^{*} & =\left\{\boldsymbol{z} \in \boldsymbol{R}^{n d}:\langle\boldsymbol{z}-\boldsymbol{x}, \boldsymbol{y}-\boldsymbol{x}\rangle \leqq 0, \forall \boldsymbol{y} \in C(\boldsymbol{x}, \boldsymbol{l}, \alpha)\right\} \\
& =\left\{\boldsymbol{z} \in \boldsymbol{R}^{n d}:\langle\boldsymbol{z}-\boldsymbol{x},-\boldsymbol{l}\rangle \geqq \sqrt{1-\alpha^{2}}|\boldsymbol{z}-\boldsymbol{x}|\right\} .
\end{aligned}
$$

From (ii) of Lemma 3.1 and (3.2)

$$
\boldsymbol{x}-\boldsymbol{n}_{\boldsymbol{i}} \in C(\boldsymbol{x}, \boldsymbol{l}, \alpha)^{*}, \quad \forall(i, j) \in L_{\boldsymbol{x}},
$$

and hence

$$
\left\langle\boldsymbol{n}_{i j}, \boldsymbol{l}\right\rangle \geqq \sqrt{1-\alpha^{2}}, \quad \forall(i, j) \in L_{\boldsymbol{x}} .
$$


Now let $\boldsymbol{n} \in \mathscr{N}_{\boldsymbol{x}}^{\prime}$ be expressed as

$$
\boldsymbol{n}=\sum_{(i, j) \in L} c_{i j} n_{i j}, \quad c_{i j} \geqq 0 .
$$

Then by (3.3)

$$
1 \geqq\langle\boldsymbol{n}, \boldsymbol{l}\rangle=\sum_{(i, j) \in L} c_{i j}\left\langle\boldsymbol{n}_{i j}, \boldsymbol{l}\right\rangle \geqq \sqrt{1-\alpha^{2}} \sum_{(i, j) \in L} c_{i j}
$$

and hence

$$
\frac{1}{2 r_{0}}=\frac{1}{\sqrt{2} \rho \sqrt{1-\alpha^{2}}} \geqq \frac{1}{\sqrt{2} \rho} \sum_{(i, j) \in L} c_{i j} .
$$

Therefore, for any $\boldsymbol{y} \in \bar{D}$

$$
\begin{aligned}
& \langle\boldsymbol{y}-\boldsymbol{x}, \boldsymbol{n}\rangle+\frac{1}{2 r_{0}}|\boldsymbol{x}-\boldsymbol{y}|^{2} \\
& \geqq \sum_{(i, j) \in L} c_{i j}\left\langle\boldsymbol{y}-\boldsymbol{x}, \boldsymbol{n}_{i j}\right\rangle+\frac{1}{\sqrt{2} \rho} \sum_{(i, j) \in L} c_{i j}|\boldsymbol{x}-\boldsymbol{y}|^{2} \\
& =\sum_{(i, j) \in L} c_{i j}\left\{\left\langle\boldsymbol{y}-\boldsymbol{x}, \boldsymbol{n}_{i j}\right\rangle+\frac{1}{\sqrt{2} \rho}|\boldsymbol{x}-\boldsymbol{y}|^{2}\right\} \geqq 0
\end{aligned}
$$

by (ii) of Lemma 3.1 and Remark 1.1. The proof of Lemma 3.2 is finished.

Lemma 3.3. For any $\varepsilon(0<\varepsilon<1)$ and $x \in \partial D$ there exists $\delta^{\prime}>0$ such that

$$
\left\{\bigcap_{(i, j) \in L} C_{i j}(\boldsymbol{x}, \varepsilon)\right\} \cap B\left(\boldsymbol{x}, \delta^{\prime}\right) \subset D \cup\{x\},
$$

where

$$
C_{i j}(\boldsymbol{x}, \varepsilon)=\left\{\boldsymbol{y} \in \boldsymbol{R}^{n d}:\left\langle\boldsymbol{y}-\boldsymbol{x}, \boldsymbol{n}_{i j}\right\rangle \geqq \varepsilon|\boldsymbol{y}-\boldsymbol{x}|\right\},(i, j) \in L .
$$

Proof. Let $\boldsymbol{y} \in C_{i j}(\boldsymbol{y}, \varepsilon), \boldsymbol{y} \neq \boldsymbol{x}$. Then $\boldsymbol{y}$ can be expressed as

$$
\boldsymbol{y}=\boldsymbol{x}+\boldsymbol{z},\left\langle\boldsymbol{z}, \boldsymbol{n}_{i j}\right\rangle \geqq \varepsilon|\boldsymbol{z}|>0,
$$

and hence

$$
\begin{aligned}
\left|y_{i}-y_{j}\right|^{2} & =\left|x_{i}-x_{j}\right|^{2}+2\left\langle x_{i}-x_{j}, z_{i}-z_{j}\right\rangle+\left|z_{i}-z_{j}\right|^{2} \\
& \geqq \rho^{2}+2\left\langle z_{i}-z_{j}, x_{i}-x_{j}\right\rangle>\rho^{2},
\end{aligned}
$$

because

$$
\begin{aligned}
0\left\langle\left\langle z, n_{i j}\right\rangle\right. & =\frac{1}{\sqrt{2} \rho}\left\langle z_{i}, x_{i}-x_{j}\right\rangle+\frac{1}{\sqrt{2} \rho}\left\langle z_{j}, x_{j}-x_{i}\right\rangle \\
& =\frac{1}{\sqrt{2} \rho}\left\langle z_{i}-z_{j}, x_{i}-x_{j}\right\rangle
\end{aligned}
$$


Therefore $C_{i j}(\boldsymbol{x}, \varepsilon) \subset D_{i j} \cup\{\boldsymbol{x}\}$, and hence

$$
\bigcap_{(i, j) \in L} C_{i j}(\boldsymbol{x}, \varepsilon) \subset\left\{\bigcap_{(i, j) \in L} D_{i j}\right\} \cup\{\boldsymbol{x}\} .
$$

Since $\left|x_{i}-x_{j}\right|>\rho$ for any $(i, j) \notin L_{x}$, there exists $\delta^{\prime}>0$ such that for any $\boldsymbol{y} \in$ $B\left(\boldsymbol{x}, \delta^{\prime}\right)$

$$
\left|y_{i}-y_{j}\right|>\rho \text { for any }(i, j) \notin L_{x} .
$$

This combined with (3.4) implies

$$
\left\{\bigcap_{(i, j) \in L} C_{i j}(\boldsymbol{x}, \varepsilon)\right\} \cap B\left(\boldsymbol{x}, \delta^{\prime}\right) \subset D \cup\{\boldsymbol{x}\} .
$$

The proof of the lemma is finished.

Proof of Proposition 3.1. We make use of Lemma 3.3 and then Corollary in $[1: \mathrm{p} .11]$ to obtain

$$
\boldsymbol{x}-\mathscr{N}_{x} \subset\left\{\bigcap_{(i, j) \in L} C_{i j}(\boldsymbol{x}, \varepsilon)\right\}^{*}=\overline{\sum_{(i, j) \in L} C_{i j}(\boldsymbol{x}, \varepsilon)^{*}},
$$

where $\Sigma$ means the vector sum. Thus

$$
\boldsymbol{x}-\mathscr{N}_{\boldsymbol{x}} \subset \bigcap_{0<\varepsilon<1}\left\{\overline{\left.\sum_{(i, j) \in L} C_{i j}(\boldsymbol{x}, \varepsilon)^{*}\right\}} .\right.
$$

But the right-hand side of the above is the convex cone with vertex $\boldsymbol{x}$ spanned by $\left\{\boldsymbol{x}-\boldsymbol{n}_{\boldsymbol{i}},(i, j) \in L_{\boldsymbol{x}}\right\}$, so we have

$$
\Re_{x} \subset \Re_{x}^{\prime}
$$

which combined with Lemma 3.2 implies

$$
\Re_{x}=\Re_{x, r_{0}}=\Re_{x}^{\prime}
$$

This means that Proposition 3.1 holds.

\section{Mutually reflecting Brownian balls}

Since the domain $D$ satisfies Conditions $(\mathrm{A})$ and $\left(\mathrm{B}^{\prime}\right)$ (and hence (B) by Remark 1.2), Theorem 1.1 guarantees the existence and uniqueness of the solution of Skorohod's equation for $D$ :

$$
\xi(t)=w(t)+\int_{0}^{t} \boldsymbol{n}(s) d \phi(s)
$$

where $w=\left(w_{1}, \cdots, w_{n}\right), w_{k} \in W, 1 \leqq k \leqq n$, and $\left|w_{i}(0)-w_{j}(0)\right| \geqq \rho, 1 \leqq i<j \leqq n . \quad A$ solution of $(4.1)$ is a pair $(\xi, \phi)$ of functions satisfying (4.1), (1.2), (1.3) and (1.4). The component-wise expression of (4.1) is 


$$
\xi_{k}(t)=w_{k}(t)+\int_{0}^{t} n_{k}(s) d \phi(s), 1 \leqq k \leqq n .
$$

In this section we prove the following theorem by showing that (4.2) is equivalent to the equation

$$
\xi_{k}(t)=w_{k}(t)+\sum_{j=1(\neq k)}^{n} \int_{0}^{t}\left(\xi_{k}(s)-\xi_{j}(s)\right) d \phi_{k j}(s), \quad 1 \leqq k \leqq n .
$$

Theorem 4.1. There exists a unique solution of Skorohod's equation (2) for the motion of mutually reflecting Brownian balls.

Proof. By Proposition 3.1 we have

$$
\begin{gathered}
n(s)=\sum_{1 \leqq i<j \leqq n} c_{i j}(s) n_{i j}(\xi(s)), \\
n_{i j}(\xi(s))=\left(0, \cdots, 0, \frac{\xi_{i}(s)-\xi_{j}(s)}{\sqrt{2} \rho}, 0, \cdots, 0, \frac{\xi_{j}(s)-\xi_{i}(s)}{\sqrt{2} \rho}, 0, \cdots, 0\right), \\
(j-\text { th }) \\
c_{i j}(s) \geqq 0,1 \leqq i<j \leqq n \\
c_{i j}(s)=0 \text { for }(i, j) \notin L_{\xi(s)} .
\end{gathered}
$$

The component-wise expression of $\boldsymbol{n}(s)$ is

$$
n_{k}(s)=\sum_{j: j>k} c_{k j}(s) \frac{\xi_{k}(s)-\xi_{j}(s)}{\sqrt{2} \rho}+\sum_{i: i<k} c_{i k}(s) \frac{\xi_{k}(s)-\xi_{i}(s)}{\sqrt{2} \rho} .
$$

Therefore if we define $c_{i j}(s)$ for $i>j$ by $c_{i j}(s)=c_{j i}(s)$, then we have

$$
n_{k}(s)=\sum_{j: j \neq k} c_{k j}(s) \frac{\xi_{k}(s)-\xi_{j}(s)}{\sqrt{2} \rho},
$$

and hence

$$
\xi_{k}(t)=w_{k}(t)+\sum_{j: j \neq k} \int_{0}^{t} c_{k j}(s) \frac{\xi_{k}(s)-\xi_{j}(s)}{\sqrt{2} \rho} d \phi(s)
$$

So if we set

$$
\phi_{k j}(t)=\frac{1}{\sqrt{2} \rho} \int_{0}^{t} c_{k j}(s) d \phi(s),
$$

we have

$$
\phi_{k j}(t)=\phi_{j k}(t),
$$




$$
\phi_{k j}(t)=\int_{0}^{t} \mathbf{1}_{\rho}\left(\left|\xi_{k}(s)-\xi_{j}(s)\right|\right) d \phi_{k j}(s)
$$

because $\phi_{k j}(t)$ can increase only when $\left|\xi_{k}(t)-\xi_{j}(t)\right|=\rho$ by (4.3d). Thus (4.1) finally yields (2). Recalling (3.1), we see easily that (2) also implies (4.1) with

$$
\begin{gathered}
\boldsymbol{n}(t)=\sum_{1 \leqq i<j \leqq n} a_{i j}(t) \boldsymbol{n}_{i j}(\xi(t)) /\left|\sum_{1 \leqq i<j \leqq n} a_{i j}(t) \boldsymbol{n}_{i j}(\xi(t))\right|, \\
d \phi(t)=\sqrt{2} \rho\left|\sum_{1 \leqq i<j \leqq n} a_{i j}(t) \boldsymbol{n}_{i j}(\xi(t))\right| d \bar{\phi}(t),
\end{gathered}
$$

where $\bar{\phi}(t)=\sum_{1 \leqq i<j \leqq n} \phi_{i j}(t)$ and $a_{i j}(t)$ is the Radon-Nikodym derivative of $d \phi_{i j}(t)$ with respect to $d \bar{\phi}(t)$. The proof of Theorem 4.1 is finished.

\section{Skorohod's SDE for mutually reflecting diffusion balls}

Let $B_{i}(t), 1 \leqq i \leqq n$, be independent $d$-dimensional $\mathscr{T}_{t}$-Brownian motions with $B_{i}(0)=0$ defined on a probability space $(\Omega, \mathscr{F}, P)$ with a filtration $\left\{\mathscr{F}_{t}\right\}_{t \geq 0}$. We assume that each $\mathscr{F}_{t}$ contains all $P$-null sets and $\mathscr{F}_{t}=\bigcap_{\varepsilon>0} \mathscr{F}_{t+\varepsilon}$. Given the coefficients

$$
\sigma: \boldsymbol{R}^{d} \rightarrow \boldsymbol{R}^{d} \otimes \boldsymbol{R}^{d}, \quad b: \boldsymbol{R}^{d} \rightarrow \boldsymbol{R}^{d},
$$

we consider the following Skorohod's SDE for mutually reflecting diffusion balls:

$$
\begin{aligned}
& d X_{i}(t)=\sigma\left(X_{i}(t)\right) d B_{i}(t)+b\left(X_{i}(t)\right) d t \\
& \quad+\sum_{j=1(\neq i)}^{n}\left(X_{i}(t)-X_{j}(t)\right) d \Phi_{i j}(t), \quad 1 \leqq i \leqq n,
\end{aligned}
$$

where the initial values are assumed to be $\mathscr{F}_{0}$-measurable random variables satisfying $\left|X_{i}(0)-X_{j}(0)\right|>\rho, 1 \leqq i<j \leqq n$. The solution $X_{i}(t), 1 \leqq i \leqq n$, should be found under the following conditions.

(5.2) $X_{i}(t)$ 's are $\mathscr{F}_{t}$-adapted continuous processes with $\left|X_{i}(t)-X_{j}(t)\right| \geqq \rho$, $1 \leqq i<j \leqq n, t \geqq 0$.

(5.3) $\Phi_{i j}(t)$ 's are $\mathscr{F}_{t}$-adapted continuous non-decreasing processes with $\Phi_{i j}(0)$ $=0, \Phi_{i j}(t)=\Phi_{j i}(t)$ and

$$
\Phi_{i j}(t)=\int_{0}^{t} \mathbf{1}_{\rho}\left(\left|X_{i}(s)-X_{j}(s)\right|\right) d \Phi_{i j}(s) \text {. }
$$

In this section we prove the following theorem.

Theorem 5.1. Suppose $\sigma$ and $b$ are bounded and Lipschitz continuous. Then there exists a unique strong solution of (5.1).

Proof. Let 


$$
D=\left\{\boldsymbol{x}=\left(x_{1}, \cdots, x_{n}\right) \in \boldsymbol{R}^{n d}:\left|x_{i}-x_{j}\right|>\rho, 1 \leqq i<j \leqq n\right\},
$$

and for $\boldsymbol{x}=\left(x_{1}, \cdots, x_{n}\right) \in \boldsymbol{R}^{n d}$ set

$$
\boldsymbol{\sigma}(\boldsymbol{x})=\left[\begin{array}{cccc}
\sigma\left(x_{1}\right) & & & 0 \\
& \sigma\left(x_{2}\right) & & \\
& & \ddots & \\
0 & & & \sigma\left(x_{n}\right)
\end{array}\right], \quad \boldsymbol{b}(\boldsymbol{x})=\left[\begin{array}{c}
b\left(x_{1}\right) \\
\vdots \\
b\left(x_{n}\right)
\end{array}\right] .
$$

Then as in 4 the $\operatorname{SDE}(5.1)$ can be regarded as Skorohod's SDE for $D$

$$
d \boldsymbol{X}(t)=\boldsymbol{\sigma}(\boldsymbol{X}(t)) d \boldsymbol{B}(t)+\boldsymbol{b}(\boldsymbol{X}(t)) d t+\boldsymbol{n}(t) d \Phi(t),
$$

where $\boldsymbol{B}(t)$ is an $n d$-dimensional $\mathscr{F}_{t}$-Brownian motion. The solution $\boldsymbol{X}(t)$ is to be found under the following conditions.

(5.5) $\boldsymbol{X}(t)$ is a $\bar{D}$-valued continuous process.

(5.6) $\boldsymbol{n}(t) \in \mathscr{T}_{X(t)}$ if $\boldsymbol{X}(t) \in \partial D$.

(5.7) $\Phi(t)$ is a continuous non-decreasing process and

$$
\Phi(t)=\int_{0}^{t} \mathbf{1}_{\partial D}(X(s)) d \Phi(s)
$$

But since $D$ satisfies Conditions (A) and (B), it follows immediately from Theorem 5.1 in [6] that the Skorohod's SDE (5.4) has a unique strong solution. The proof is finished.

\section{References}

[1] W. Fenchel: Convex cones, sets, and functions, Lecture notes, Princeton Univ., 1953.

[2] H. Frankowska: A viability approach to the Skorohod problem, Stochastics 14 (1985), 227-244.

[3] N. Ikeda and S. Watanabe: Stochastic differential equations and diffusion processes, North Holland-Kodansha, Amsterdam-Tokyo, 1981.

[4] P.L. Lions and A.S. Sznitman: Stochastic differential equations with reflecting boundary conditions, Comm. Pure Appl. Math. 37 (1984), 511-537.

[5] H.P. McKean: Stochastic integrals, Academic Press, New York, 1969.

[6] Y. Saisho: Stochastic differential equations for multi-dimensional domain with reflecting boundary, to appear in Probab. Th. Rel. Fields.

[7] A.V. Skorohod: Stochastic equations for diffusion processes in a bounded region 1, 2, Theor. Veroyatnost. i Primenen. 6 (1961), 264-274; 7 (1962), 3-23.

[8] H. Tanaka: Stochastic differential equations with reflecting boundary condition in 
convex regions, Hiroshima Math. J. 9 (1979), 163-177.

Department of Mathematics Faculty of Science and Technology Keio University

Kohokuku, Yokohama 223

Japan 